\title{
Papers
}

\section{Evaluation of WHO criteria for identifying patients with severe acute respiratory syndrome out of hospital: prospective observational study}

Timothy H Rainer, Peter A Cameron, DeVilliers Smit, Kim L Ong, Alex Ng Wing Hung, David Chan Po Nin, Anil T Ahuja, Louis Chan Yik Si, Joseph J Y Sung

Accident and

Emergency

Medicine Academic

Unit, Chinese

University of Hong

Kong, Shatin, New

Territories, Hong

Kong, China

Timothy H Rainer

associate professor

Peter A Cameron

professor and director

DeVilliers Smit

assistant professor

Kim L Ong

associate professor

Alex Ng Wing

Hung

medical officer

David Chan Po Nin

medical officer

Anil T Ahuja

professor

Louis Chan Yik Si

medical officer

Joseph J Y Sung

professor

Correspondence to:

T H Rainer,

Department of

Emergency

Medicine, Prince of

Wales Hospital,

Shatin, New

Territories, Hong

Kong, China

rainer1091@

cuhk.edu.hk

BMJ 2003;326:1354-8

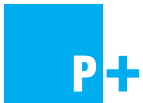

Details of health advice given to attenders at the screening clinic are on bmi.com

\begin{abstract}
Objectives To determine the clinical and radiological features of severe acute respiratory syndrome (SARS) and to evaluate the accuracy of the World Health Organization's guidelines on defining cases of SARS. Design Prospective observational study.

Setting A newly set up SARS screening clinic in the emergency department of a university hospital in Hong Kong's New Territories.

Participants 556 hospital staff, patients, and relatives who attended the screening clinic and who had had contact with someone with SARS.

Main outcome measure Number of confirmed cases of SARS.

Results Of the 556 people, 141 were admitted to hospital, and 97 had confirmed SARS. Fever, chills, malaise, myalgia, rigor, loss of appetite, vomiting, diarrhoea, and neck pain but not respiratory tract symptoms were significantly more common among the 97 patients than among the other patients. The overall accuracy of the WHO guidelines for identifying suspected SARS was $83 \%$ and their negative predictive value was $86 \%$ (95\% confidence interval $83 \%$ to $89 \%$ ). They had a sensitivity of $26 \%$ ( $17 \%$ to $36 \%)$ and a specificity of $96 \%$ (93\% to $97 \%)$. Conclusions Current WHO guidelines for diagnosing suspected SARS may not be sufficiently sensitive in assessing patients before admission to hospital. Daily follow up, evaluation of non-respiratory, systemic symptoms, and chest radiography would be better screening tools.
\end{abstract}

\section{Introduction}

Initial reports on severe acute respiratory syndrome (SARS) described the clinical features of confirmed cases. ${ }^{1-4}$ Later reports have described the epidemiology and progression of the illness in greater detail. ${ }^{56}$ On the basis of early findings in hospitals, the World Health Organization and the Hospital Authority of Hong Kong produced case definitions for suspected and probable cases of SARS that may be used for screening patients before admission to hospital and in non-clinical contexts such as airports. ${ }^{78}$ The discovery of the virus and the development of rapid serological tests may improve case definition, but the tests are not yet widely available..$^{9-11}$

In the first two weeks of March 2003, 15 doctors, 15 nurses, 17 medical students, and five other staff (auxiliary staff, a clerk, and cleaning staff) associated with ward $8 \mathrm{~A}$ of the Prince of Wales Hospital were infected with SARS. In response to this outbreak the hospital set up an emergency screening clinic on 12 March to evaluate all staff and their immediate contacts. The clinic gave us the opportunity to study the clinical response to the virus in a high contact environment. We investigated the clinical features of SARS in the early stages of infection to evaluate the WHO criteria for identifying suspected and probable cases of SARS and to report the safety of our current strategies to prevent the spread of SARS among our staff.

\section{Methods}

The study was conducted from 12 March to 31 March 2003 in the newly opened SARS clinic in the emergency department of the Prince of Wales Hospital, a 1400 bed university teaching hospital in the New Territories of Hong Kong. Health advice was given to all hospital staff, patients, and relatives who attended the clinic (see bmj.com for details).

\section{Defining cases of SARS}

As no diagnostic investigations for SARS were available at the time the clinic opened, we based diagnosis on exclusion of other diseases and on the WHO guidelines (box). ${ }^{72}$ For suspected cases, we took a broad interpretation of the respiratory symptoms in the WHO criteria to include upper and lower tract clinical features. We confirmed a diagnosis of SARS when a patient was known to have contact with someone with SARS, had documented persistent fever $\left(>38^{\circ} \mathrm{C}\right)$, a consistent clinical course of the illness, and evidence of pneumonia.

We used plain radiography or computed tomography to diagnose pneumonia. We diagnosed non-SARS pneumonia if the patient responded well to antibiotics within 48 hours. Final diagnoses were made by a team of general medical, respiratory, and infectious diseases clinicians. The recent discovery of the virus and the development of an immunofluorescence assay based 
WHO case definitions for suspected and probable SARS

SARS is suspected in patients with:

- High fever $\left(>38^{\circ} \mathrm{C}\right)$

- One or more respiratory symptoms (such as cough, shortness of breath, or breathing difficulty), and

- Close contact with a person previously diagnosed with SARS (having cared for, lived with, or had direct contact with bodily secretions of a person with SARS)

SARS is probable when a patient meets the criteria of a suspected case and there is radiological evidence of infiltrates consistent with pneumonia or respiratory distress syndrome.

on vero cells infected with coronavirus have since allowed us to confirm diagnoses by measuring levels of anti-coronavirus IgG antibody in saved serum samples.

\section{Inclusion and exclusion criteria}

All hospital staff, patients, and relatives of staff or patients had access to the clinic. People attending the clinic were included in the study if they had had contact with anyone with SARS. We excluded children aged less than 11 years because their laboratory results and the clinical course of the disease are likely to differ from those of adults. Patients admitted to hospital with pneumonia but who had a diagnosis of non-SARS pneumonia were not excluded from the analysis.

\section{Discharge and follow up criteria}

Patients were discharged after their first attendance at the clinic if they had vague or no symptoms, no fever, and normal radiological and laboratory test results. These patients were given hygiene advice and told to return if they became feverish. Patients were followed up daily after their first attendance at the clinic if they had had contact with someone with SARS, had one or more symptoms (upper and lower respiratory tract symptoms, gastrointestinal symptoms, or systemic symptoms), were feverish $\left(>38^{\circ} \mathrm{C}\right)$ on at least one occasion, and had a normal or indeterminate chest radiograph and if the results of investigations were abnormal (such as leucopenia, lymphopenia, monocytosis, or thrombocytosis). These patients were clinically assessed and had anteroposterior chest radiography daily. Patients were given hygiene advice and a follow up appointment for the next day. Patients who were followed up daily and who were clear of symptoms for 48 hours, with no documented fever and normal chest radiographs and laboratory tests, were discharged.

\section{Data collection and measurement}

All patients completed a health questionnaire and saw a doctor. Basic observations were recorded, including pulse, systolic and diastolic blood pressure, respiratory rate, tympanic temperature, and oxygen saturation in room air. All patients had daily frontal, plain chest radiography until either their symptoms subsided or a pneumonic change was seen. Patients whose fever and symptoms persisted for more than two days underwent standard and high resolution computed tomography, even if their chest radiographs were normal, to confirm or exclude occult pneumonia. Chest radiographs were evaluated firstly by a specialist emergency physician with reference to clinical details and then by a radiologist without reference to details. The primary clinical outcome was confirmed cases of SARS.

\section{Statistical analysis}

We used the unpaired Student's $t$ test to analyse continuous data and the $\chi^{2}$ test or Fisher's exact test for categorical data. We used Statview for Windows version 5.0 (Abacus Concepts, SAS Institute, Cary, NC). All analyses were two tailed. $\mathrm{P}$ values of $<0.05$ were considered statistically significant.

\section{Results}

Between 11 March and 31 March 2003 a total of 556 people with a history of contact with someone with

Table 1 Characteristics of patients presenting to the SARS screening clinic who had previous contact with someone with SARS. Values are numbers (percentage) unless otherwise stated

\begin{tabular}{|c|c|c|c|c|}
\hline Characteristic & $\begin{array}{l}\text { All patients } \\
(\mathrm{n}=556)\end{array}$ & $\begin{array}{l}\text { Patients without SARS } \\
\qquad(\mathrm{n}=459)\end{array}$ & $\begin{array}{l}\text { Patients with SARS } \\
\qquad(\mathrm{n}=97)\end{array}$ & $P$ value* \\
\hline Mean (SD) age (years) & $35.8(14.0)$ & $35.6(13.7)$ & $37.0(15.4)$ & 0.73 \\
\hline No $(\%)$ of men & $168(30)$ & $131(29)$ & $37(38)$ & 0.06 \\
\hline $\begin{array}{l}\text { Median No of days (interquartile; range) between onset } \\
\text { of symptoms and first presentation }\end{array}$ & 3.0 (1.0 to $5.0 ; 1$ to 30 ) & 3.0 (1.0 to $5.0 ; 1$ to 30 ) & 3.0 (1.75 to $4.25 ; 1$ to 15$)$ & 0.22 \\
\hline \multicolumn{5}{|l|}{ Status: } \\
\hline Healthcare worker & $325(59)$ & $262(57)$ & $63(65)$ & 0.01 \\
\hline Other hospital staff & $82(15)$ & $77(17)$ & $5(5)$ & \\
\hline Patients and relatives of staff or patients & $149(27)$ & $120(26)$ & $29(30)$ & \\
\hline \multicolumn{5}{|l|}{ Presentation: } \\
\hline Without symptoms & $41(7)$ & $41(9)$ & - & 0.0022 \\
\hline With symptoms & $515(93)$ & 418 (91) & $97(100)$ & \\
\hline Patients who met WHO definitions for suspected SARS & $46(8)$ & $21(5)$ & $25(26)$ & $<0.0001$ \\
\hline \multicolumn{5}{|l|}{ Disposal: } \\
\hline Follow up without admission & $374(67)$ & $372(81)$ & $2(2)$ & $<0.0001$ \\
\hline Admission to hospital & $141(25)$ & $46(10)$ & $95(98)$ & \\
\hline Admission at first presentation & $79(14)$ & $33(7)$ & $46(47)$ & \\
\hline Admission during follow up & $62(11)$ & $13(3)$ & $49(51)$ & \\
\hline \multicolumn{5}{|l|}{ Final diagnosis: } \\
\hline Pneumonia† & $114(21)$ & $17(4)$ & $97(100)$ & $<0.0001$ \\
\hline Upper respiratory tract infection & $81(15)$ & $81(18)$ & - & \\
\hline
\end{tabular}

*Student's $t$ test or $\chi^{2}$ test.

†Typical pneumonia was diagnosed in 17 patients who recovered quickly after treatment with antibiotics alone. 
Table 2 Clinical characteristics of people presenting to screening clinic with symptoms. Values are numbers (percentage) of patients

\begin{tabular}{|c|c|c|c|c|}
\hline Characteristic & Total $(n=515)$ & Patients without SARS $(n=418)$ & Patients with SARS ( $\mathrm{n}=97$ ) & $P$ value* \\
\hline \multicolumn{5}{|l|}{ Clinical features (№ (\%) of patients) } \\
\hline Fever & $233(45)$ & $154(37)$ & $79(81)$ & $<0.0001$ \\
\hline Chills & $139(27)$ & $87(21)$ & $52(54)$ & $<0.0001$ \\
\hline Malaise & $118(23)$ & $85(20)$ & $33(34)$ & 0.004 \\
\hline Myalgia & $76(15)$ & $50(12)$ & $26(27)$ & 0.0002 \\
\hline Rigor & $27(5)$ & $15(4)$ & $12(12)$ & 0.0005 \\
\hline Neck pain & $4(<1)$ & $1(0.2)$ & $3(3)$ & 0.004 \\
\hline Cough & $363(70)$ & $301(72)$ & $62(64)$ & 0.12 \\
\hline Sputum & $146(28)$ & $121(29)$ & $25(26)$ & 0.52 \\
\hline Sore throat & $195(38)$ & $161(39)$ & $34(35)$ & 0.53 \\
\hline Runny nose & $161(31)$ & $136(33)$ & $25(26)$ & 0.20 \\
\hline Chest pain & $5(1)$ & $4(1)$ & $1(1)$ & 0.95 \\
\hline Shortness of breath & $40(8)$ & $28(7)$ & $12(12)$ & 0.04 \\
\hline Loss of appetite & $9(2)$ & $4(1)$ & $5(5)$ & 0.005 \\
\hline Vomiting & $15(3)$ & $9(2)$ & $6(6)$ & 0.03 \\
\hline Abdominal pain & $11(2)$ & 7 (2) & $4(4)$ & 0.14 \\
\hline Diarrhoea & $19(4)$ & $12(3)$ & $7(7)$ & 0.04 \\
\hline Night sweats & $3(<1)$ & $2(0.5)$ & $1(1)$ & 0.52 \\
\hline Anorexia & $5(1)$ & $5(1)$ & $0(0)$ & 0.28 \\
\hline Headache & $110(21)$ & $85(20)$ & $25(26)$ & 0.24 \\
\hline Dizziness & $28(5)$ & $22(5)$ & $6(6)$ & 0.72 \\
\hline Rash & 0 & 0 & 0 & \\
\hline \multicolumn{5}{|l|}{ Basic observations (mean (SD)) } \\
\hline Heart rate per minute & $95.2(17.4)$ & $94.0(17.0)$ & $104.5(16.8)$ & $<0.0001$ \\
\hline Systolic blood pressure (mm Hg) & $136.6(19.5)$ & $137.3(19.0)$ & $132.8(20.8)$ & 0.04 \\
\hline Diastolic blood pressure $(\mathrm{mm} \mathrm{Hg})$ & $75.8(12.7)$ & $76.1(12.8)$ & $73.7(12.4)$ & 0.1 \\
\hline Respiratory rate per minute & $18.6(3.3)$ & $18.7(3.6)$ & $18.8(2.0)$ & 0.74 \\
\hline Highest temperature while at clinic & $37.0(0.9)$ & $36.8(0.8)$ & $37.9(0.8)$ & $<0.0001$ \\
\hline
\end{tabular}

${ }^{*} \chi^{2}$ test, Fisher's exact test, or Student's $t$ test

SARS attended the screening clinic (table 1). We excluded 41 patients who had no symptoms. Table 2 shows the clinical features and observations in the other 515 patients. Symptoms that were more common (though not significantly) among patients who did not develop SARS than in patients with confirmed SARS were cough (72\% of patients), sputum production (29\%), sore throat (39\%), and runny nose (33\%). Clinical symptoms that were significantly more common among patients with confirmed SARS were fever, chills, malaise, myalgia, rigor, neck pain, loss of appetite, shortness of breath, vomiting, and diarrhoea. Of the common upper and lower respiratory tract symptoms only shortness of breath was significantly more common among patients with SARS.

Only two patients with obvious radiological evidence of consolidation had chest signs that were detectable on physical examination. Compared with patients who did not develop SARS, patients with confirmed SARS had a significantly higher heart rate, lower mean systolic blood pressure, and higher mean

Table 3 Accuracy of WHO criteria for identifying suspected severe acute respiratory syndrome (SARS)

\begin{tabular}{lrcc} 
& \multicolumn{3}{c}{ SARS confirmed (No of patients) } \\
\cline { 2 - 4 } & No & Yes & Total \\
\hline $\begin{array}{l}\text { Suspected SARS according to WHO } \\
\text { criteria }\end{array}$ & & & \\
\hline No & 438 & 72 & 510 \\
\hline Yes & 21 & 25 & 46 \\
\hline Total & 459 & 97 & 556
\end{tabular}

Sensitivity $25.8 \%$ (95\% confidence interval $17.4 \%$ to $35.7 \%$ ), specificity $95.4 \%$ (93.1\% to $97.1 \%$ ), positive predictive value $54.3 \%$ (39.0\% to $69.1 \%$ ), negative predictive value $85.9 \%(82.6 \%$ to $88.8 \%$ ). temperature. Respiratory rate did not differ between the groups.

\section{Predictive ability of the WHO criteria for} diagnosing suspected SARS

Of the 97 patients with confirmed SARS, 25 met the criteria for suspected SARS in the WHO guidelines (table 3). The criteria had an overall accuracy of $83 \%$ (463 of 556 cases correctly identified). They had a negative predictive value of $86 \%(95 \%$ confidence interval $83 \%$ to $89 \%$ ), a positive predictive value of $54 \%$ (39\% to $69 \%)$, a sensitivity of $26 \%$ ( $17 \%$ to $36 \%$ ), and a specificity of $95 \%$ (93\% to $97 \%)$. Applying the WHO criteria for suspected SARS in our group of patients would have missed 72 cases $(74 \%)$. The odds ratios of predicting SARS for particular symptoms were 12.0 (6.8 to 21.0) for fever, 1.0 (0.6 to 1.7) for cough, and 1.5 (0.7 to 3.5) for shortness of breath.

\section{Radiological changes}

All patients had chest radiography. Pneumonic change was evident in 129 patients (23\%): $72(56 \%)$ on the first presentation and $57(44 \%)$ on follow up. Chest x ray changes were unifocal (figure 1), bifocal, or diffuse. The odds ratio for radiological findings predicting SARS was 32.1 (18.0 to 57.3).

High resolution computed tomography was requested for 27 patients $(5 \%)$ who had normal chest radiographs but persistent fever and symptoms. Eighteen of the 27 scans $(67 \%)$ were positive and one was indeterminate. Figure 2 shows two patients' scans that were taken on the same day: one with a retrocardiac lesion and one with a retrodiaphragmatic lesion. The median time from onset of symptoms to identification 


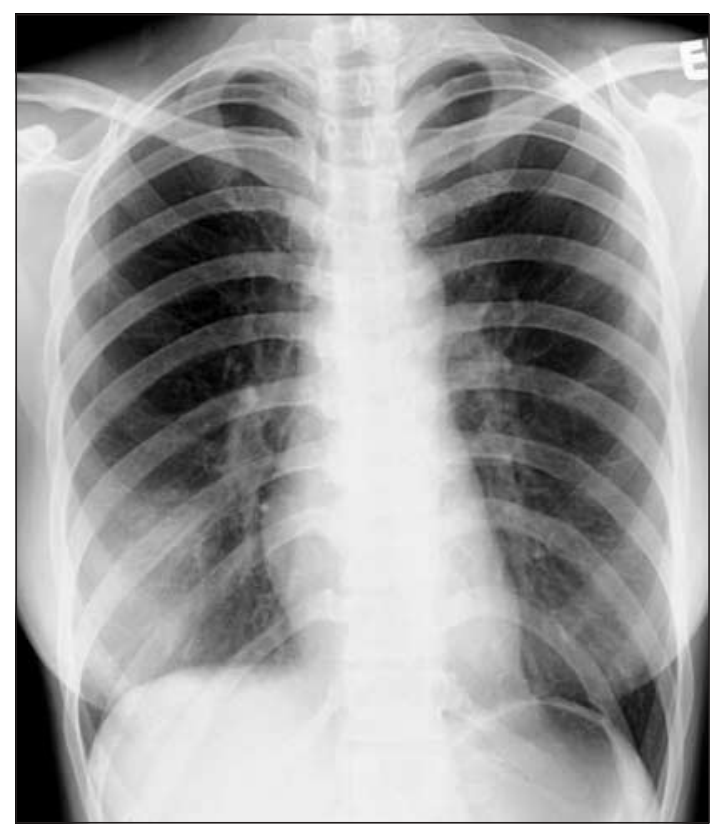

Fig 1 Frontal chest radiograph showing an area of opacification in the right lower zone

of positive radiological changes was four days and to identification of changes in scans was seven days.

\section{Secondary infections and serology}

No healthcare workers in the clinic were infected once it was fully operational, and no secondary infections occurred among the patients with suspected SARS. A preliminary serological analysis of samples from 179 patients who have attended the clinic have shown that 98 samples from 99 people with confirmed SARS were positive for coronavirus and that all the samples from 80 people who did not develop SARS were negative.

\section{Discussion}

The WHO guidelines on diagnosing SARS emphasise respiratory tract symptoms such as cough, shortness of breath, and breathing difficulty. However, these clinical symptoms in the WHO case definitions do not feature strongly in the early stages of the illness, when patients are highly infectious but before they are hospitalised. In screening patients for SARS systemic symptoms such as fever, chills, malaise, myalgia, and rigors may be better discriminators than the symptoms listed in the WHO guidelines, which were based on study of patients who were already in hospital. The absence of clinical signs in all but a few of our patients when they were screened-even in patients with obvious pneumonic changes in radiographs-means that chest radiography ought to be mandatory for all patients being screened for SARS. Of all the predictors we tested, chest radiological changes had the highest odds ratio. Almost $75 \%$ of patients in our study with history of contact with SARS and evidence of pneumonia on radiography did not have a high fever.

One limitation of our study is that it took place in a single centre with a high proportion of healthcare workers and primary contacts, and thus the results may not be generalisable to the wider community. Establishing whether patients have had contact with someone with SARS is difficult and sometimes
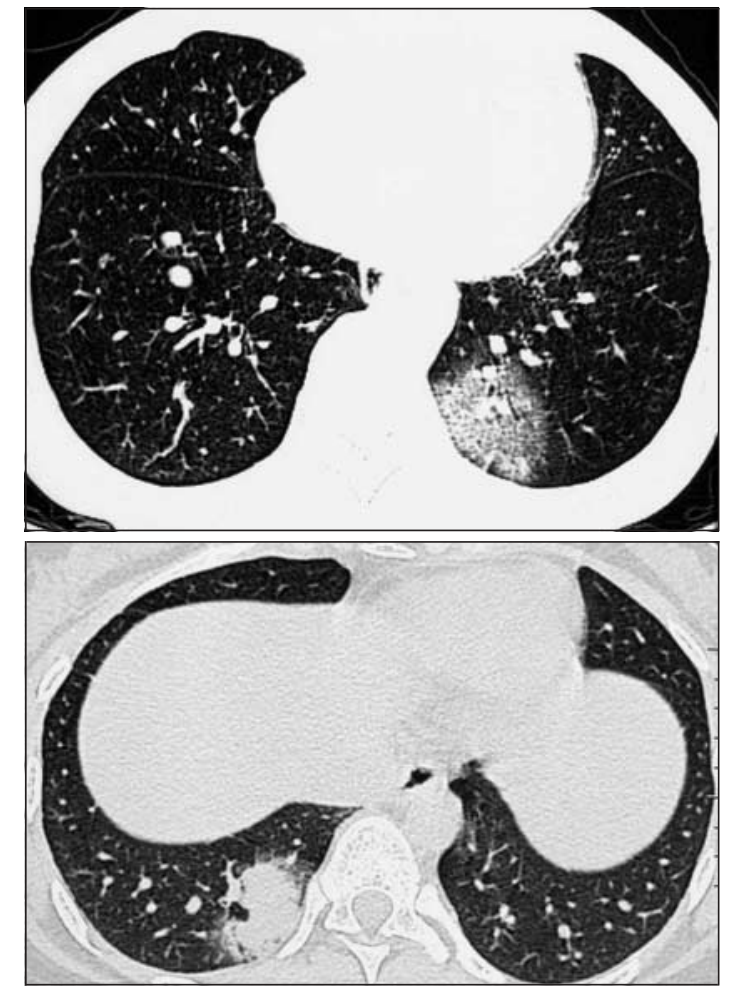

Fig 2 High resolution computed tomograms of two patients whose chest radiographs were normal: (above) ground glass opacification in the posterior segments of the left lower lobe (difficult to identify on a frontal chest radiograph because of location behind the heart); (below) ground glass opacification in the posterior segments of the right lower lobe (difficult to identify on a frontal chest radiograph because of location behind the diaphragm)

impractical. However, one advantage of our group was that contact was highly likely and was documented. Screening may be more difficult in situations where a contact history is difficult to establish.

Preliminary blood testing for coronavirus indicates that our screening and diagnostic criteria are over $99 \%$ accurate. Our patients showed no secondary infection or severe secondary deterioration, prevention of which was the main reason for setting up the screening clinic,

\section{What is already known on this topic}

The main criteria in WHO's case definitions for suspected SARS among people who have had close contact are fever $\left(>38^{\circ} \mathrm{C}\right)$ and respiratory symptoms such as cough or breathing difficulty

WHO's case definitions, which are based on study of patients in hospital, have not been evaluated in the context of screening patients before admission to hospital

\section{What this study adds}

In the early stages of SARS the main discriminating symptoms are not cough and breathing difficulty but fever, chills, malaise, myalgia, rigors, and, possibly, abdominal pain and headache

Documented fever $\left(>38^{\circ} \mathrm{C}\right)$ is uncommon in the early stages, and radiological evidence of pneumonic changes often precedes fever

WHO case definitions for suspected SARS have a negative predictive value of $85 \%$ and a sensitivity of $26 \%$ for detecting SARS in patients who have not been admitted to hospital 
and thus our protocols seem to be safe. No healthcare workers in the clinic or close contacts of the patients became infected.

As SARS continues to spread worldwide, other healthcare settings will need to screen staff and patients who have symptoms and who have had close contact with SARS patients after an outbreak. ${ }^{13}$ With a sensitivity of $26 \%$ and a negative predictive value of $85 \%$, the WHO criteria should be refined to include routine daily follow up, documentation of nonrespiratory systemic symptoms, and daily chest radiography until patients have passed at least 48 hours without symptoms.

Contributors: THR had the idea for the study, oversaw its planning and execution and the statistical analysis, and prepared the manuscript. PAC, DS, and KLO participated in the planning, execution, and analysis. ANWH, DCPN, and ATA were responsible for assessment of radiographs and scans. LCYS planned the epidemiological follow up. JJYS supervised the clinical assessment of patients after admission. All authors contributed to the final version of the paper. THR will act as guarantor.

Funding: No additional funding.

Competing interests: None declared.

1 Poutanen SM, Low DE, Henry B, Finkelstein S, Rose D, Green K, et al. Identification of severe acute respiratory syndrome in Canada. $N$ Engl J Med 2003:348:1995-2005.
2 Tsang KW, Ho PL, Ooi GC, Yee WK, Wang T, Chan-Yeung M, et al. A cluster of cases of severe acute respiratory syndrome in Hong Kong. A cluster of cases of severe acu

3 Lee N, Hui D, Wu A, Chan P, Cameron P, Joynt G, et al. A major outbreak of severe acute respiratory syndrome (SARS) in Hong Kong. $N$ Engl J Med 2003;348:1986-94

4 Chan-Yeung M, Yu WC. Outbreak of severe acute respiratory syndrome in Hong Kong Special Administrative Region: case report. BMJ 2003;326:850-2.

5 Donnelly C, Ghani AC, Leung GM, Hedley AJ, Fraser C, Riley S, et al. Epidemiological determinants of spread of causal agent of severe acute respiratory syndrome. Lancet 2003;361:1761-6.

6 Peiris JSM, Chu CM, Cheng VCC, Chan KS, Hung IFN, Pool LLM, et al. Clinical progression and viral load in a community outbreak of coronavirus-associated SARS pneumonia: a prospective study. Lancet coronavirus-associ

2003;361:1767-72.
World Health Organization. Case definitions for surveillance of severe World Health Organization. Case definitions for surveillance of severe
acute respiratory syndrome (SARS). www.who.int/csr/sars/ casedefinition/en/ (accessed 10 April 2003).

8 Ho W, the Hong Kong Hospital Authority Working Group on SARS and the Central Committee on Infection Control. Guideline on managemen of severe acute respiratory syndrome (SARS). Lancet 2003;361:1313.

9 Peiris JSM, Lai ST, Poon LLM, Guan Y, Yam LYC, Lim W, et al. Coronavirus as a possible cause of severe acute respiratory syndrome. Lancet 2003:361:1319-25.

10 Ksiazek TG, Frdman D, Goldsmith C, Zaki SR, Peret T, Emery S, et al. A novel coronavirus associated with severe acute respiratory syndrome. New Engl J Med 2003;348:1953-66.

11 Drosten C, Gunther S, Preiser W, van der Werf S, Brodt H-R, Becker S, et al. Identification of a novel coronavirus in patients with severe acute respiratory syndrome. New EnglJ Med 2003;348:1967-76.

12 World Health Organization. Severe acute respiratory syndrome. $W k l$, Epidemiol Rec 2003;78:97-120.

13 World Health Organization. Cumulative number of reported cases (SARS) from 1st November 2002 to 9th May 2003. www.who.int/csr/ sarscountry/2003 05 09/en/ (accessed 9 May 2003).

(Accepted 22 May 2003)
Department of Medicine and Therapeutics, Chinese University of Hong Kong, Prince of Wales Hospital, Shatin, New Territories, Hong Kong Special Administrative Region, China

Raymond S M Wong

haematologist

Alan Wu

medical and health

officer

Nelson Lee

medical and health

officer

David S Hui

associate professor

Gregory Cheng associate professor Joseph J Y Sung professor

continued over

BMJ 2003;326:1358-62

\section{Haematological manifestations in patients with severe acute respiratory syndrome: retrospective analysis}

Raymond S M Wong, Alan Wu, K F To, Nelson Lee, Christopher W K Lam, C K Wong, Paul K S Chan, Margaret H L Ng, L M Yu, David S Hui, John S Tam, Gregory Cheng, Joseph J Y Sung

\author{
Abstract \\ Objectives To evaluate the haematological findings \\ of patients with severe acute respiratory syndrome \\ (SARS). \\ Design Analysis of the demographic, clinical, and \\ laboratory characteristics of patients with SARS. \\ Setting Prince of Wales Hospital, Hong Kong. \\ Subjects All patients with a diagnosis of SARS \\ between 11 March and 29 March 2003 who had no \\ pre-existing haematological disorders. \\ Main outcome measures Clinical end points \\ included the need for intensive care and death. \\ Univariate and multivariate analyses were performed \\ to examine factors associated with adverse outcome. \\ Results 64 male and 93 female patients were included \\ in this study. The most common findings included \\ lymphopenia in 153 (98\%) of the 157 patients, \\ neutrophilia in $129(82 \%)$, thrombocytopenia in 87 \\ patients $(55 \%)$, followed by thrombocytosis in 77 \\ (49\%), and isolated prolonged activated partial \\ thromboplastin time in 96 patients $(63 \%)$. The \\ haemoglobin count dropped by more than $20 \mathrm{~g} / 1$ \\ from baseline in $95(61 \%)$ patients. Four patients \\ $(2.5 \%)$ developed disseminated intravascular \\ coagulation. Lymphopenia was shown in \\ haemato-lymphoid organs at postmortem
}

examination. Multivariate analysis showed that advanced age and a high concentration of lactate dehydrogenase at presentation were independent predictors of an adverse outcome. Subsets of peripheral blood lymphocytes were analysed in 31 patients. The counts of $\mathrm{CD} 4$ positive and $\mathrm{CD} 8$ positive $\mathrm{T}$ cells fell early in the course of illness. Low counts of CD4 and CD8 cells at presentation were associated with adverse outcomes.

Conclusions Abnormal haematological variables were common among patients with SARS. Lymphopenia and the depletion of T lymphocyte subsets may be associated with disease activity.

\section{Introduction}

An outbreak of severe acute respiratory syndrome (SARS) has recently been reported from Hong Kong. ${ }^{1}$ A novel coronavirus has been identified as the aetiological agent of the syndrome..$^{23}$ Viral infection may produce various haematological changes. Early studies have shown that lymphopenia and thrombocytopenia are common among patients with SARS. ${ }^{14}$ This study summarises the haematological findings in patients with SARS who were treated at the Prince of Wales Hospital, Hong Kong. 